\title{
Blood pressure in primary school children in Uganda: a cross-sectional survey
}

\author{
Farah Kidy ${ }^{1,4}$, Diana Rutebarika ${ }^{2,5}$, Swaib A Lule ${ }^{2}$, Moses Kizza ${ }^{2}$, Amos Odiit ${ }^{3,6}$, Emily L Webb \\ and Alison M Elliott ${ }^{1,2^{*}}$
}

\begin{abstract}
Background: Non-communicable diseases are an emerging concern in sub-Saharan Africa, and risks for these conditions are often based on exposures in early life, with premonitory signs developing during childhood. The prevalence of hypertension has been reported to be high in African adults, but little is known about blood pressure in African children. We studied prevalence and risk factors for high blood pressure (HBP) among school children in central Uganda.
\end{abstract}

Methods: Two urban and five rural schools were randomly selected from government schools in Wakiso district, Uganda. Questionnaires were administered and anthropometric measures taken. Blood pressure (BP) was measured three times in one sitting (on day 1) and the average compared to internationally-used normograms. Children with BP >95th percentile were re-tested at two additional sittings (day 2 and day 3) within one week, and at two further follow up visits over a period of six months. Those with sustained HBP were referred for further investigation.

Results: Of 552 students included, 539 completed the initial assessments (days 1-3) of whom 92 (17.1\%) had HBP at the initial sitting. Age (adjusted odds ratio (aOR) 1.29 (95\% confidence interval 1.14, 1.47), p<0.001), body mass index $(1.70(1.25-2.31) p=0.001)$ and soil-transmitted helminths (2.52 (1.04-6.11), 0.04) were associated with increased prevalence of HBP at the initial sitting. After further investigation, sustained HBP was seen in 14 children, yielding an estimated prevalence of 3.8\% allowing for losses to follow up. Four children required treatment.

Conclusion: It is feasible to measure blood pressure accurately in the school setting. A high HBP prevalence on initial readings gave cause for concern, but follow up suggested a true HBP prevalence commensurate with international normograms. Extended follow up is important for accurate assessment of blood pressure among African children.

Keywords: Blood pressure, Hypertension, Children, Uganda, Africa

\section{Background}

There is growing recognition of the importance of noncommunicable diseases (NCDs) in developing countries [1]. The World Health Organisation (WHO) Global Burden of Disease report states that $25 \%$ of all deaths in Africa are due to NCDs, with cardiovascular conditions contributing nearly half those deaths [2]. It is estimated that 77.1 million women and 73.6 million men in sub-Saharan Africa will be hypertensive by 2025 [3]. Sustained elevated blood pressure (BP) is a major risk

\footnotetext{
* Correspondence: alison.elliott@|shtm.ac.uk

'London School of Hygiene and Tropical Medicine, Keppel Street, London WC1E 7HT, UK

${ }^{2}$ MRC/UVRI Uganda Research Unit on AIDS, P.O. Box 49, Entebbe, Uganda Full list of author information is available at the end of the article
}

factor for stroke, coronary heart disease and renal disease. Post-mortem studies have shown that high blood pressure (HBP) in childhood, especially high systolic BP, is a risk factor for the development of fatty streaks and fibrous plaques in the coronary arteries of young people [4]. Longitudinal studies following children from age seven to young adulthood have demonstrated that high systolic and diastolic readings in childhood are predictive of hypertension in adulthood [5].

There have been limited attempts to estimate the prevalence of childhood HBP in Africa [6,7]. A recent review of HBP demonstrated prevalences ranging from $7.5 \%$ to $22.3 \%$ in data largely from South Africa [6]. Although secondary HBP (resulting from pathological insults) constitutes a 
higher proportion of HBP cases in children than in adults, primary HBP is still commoner than secondary HBP in children in developed countries, with obesity playing a major role [8]. The relative contribution of primary and secondary HBP in children in developing countries is unknown.

This school-based, cross-sectional survey, conducted in Uganda, addressed the procedures required to assess blood pressure accurately in primary school children in this setting, and investigated risk factors for high blood pressure associated with rural or urban environments: parasitic infections, body mass index (BMI) and other risk factors.

\section{Methods}

This cross-sectional study was conducted in Entebbe Municipality and Ssisa sub-country, Wakiso district, Uganda in 2010. In Uganda, in 2010 [9], school enrolment in our target age group was estimated at $84 \%$, with 4.4 million boys and 4.3 million girls enrolled. Entebbe Municipality is a relatively urbanised community close to Uganda's international airport and the capital, Kampala. The socioeconomic background of the inhabitants ranges from wealthy commuters to poor fishing communities. Ssisa sub-county comprises predominantly rural communities.

Schools in Entebbe Municipality (purposively selected to represent a relatively urban community) and in Sissa subcounty (purposively selected to represent a rural community) provided the sampling frame for the study. Only schools receiving government support were included in the sampling frame, armed forces schools and one school catering exclusively for special needs children were excluded. Schools were then selected at random from the resulting list of eligible schools. Two urban schools were selected from a total of nine eligible schools, and five rural schools, also from a total of nine eligible schools. In selected schools, all children in primary classes three, four and five were eligible for inclusion (except in one rural school, where, on the advice of the head teacher, children in primary classes two, three and four were included as likely to be more similar in age to the other schools). These classes were chosen as children were thought to be old enough to answer questionnaires and participate in the activities of the project, but avoided disruption to the highest classes, preparing for national examinations. No exclusion criteria were applied to children within the classes.

Ethical approval was obtained from the Science and Ethics Committee of the Uganda Virus Research Institute, the Uganda National Council for Science and Technology and the London School of Hygiene and Tropical Medicine. Informed written consent was obtained from parents of all participants in English or Luganda. Children also provided assent.
In the urban schools, ten boys and ten girls from each class were randomly selected to be tested for malaria and helminth infections by taking a systematic sample from the list of children for whom consent and assent had been obtained. In the smaller rural schools all participants were tested.

Data and samples were collected at an initial set of visits on three separate days ("day 1", "day 2", "day 3"; all within one week) in July or September 2010 and then at two subsequent single day follow-up visits at intervals of approximately three months ("follow up 1" and "follow up 2").

The team received training for a period of one week in the use of the equipment, issues pertaining to questionnaire delivery and confidentiality. During the initial set of visits, age, gender and tympanic temperature were recorded. Height was measured to the nearest millimetre using a measuring pole. Weight was measured to the nearest 0.1 kilogram using electronic scales, without shoes or heavy clothing. Body mass index (BMI - weight/ height ${ }^{2}$ ), BMI-for-age percentile and $\mathrm{z}$-score, and heightfor-age percentile and $\mathrm{z}$-score were calculated using WHO Anthroplus version 1.0.3. Most children were not aware of their date of birth; these children were designated to be their reported age plus 6 months.

A questionnaire, designed to obtain simple information on the child's health, and risk factors for HBP, was administered to all participants in English or Luganda.

Blood pressure was measured on the right arm using an Omron M6 automated upper arm blood pressure monitor with appropriate cuff size for the child's arm [10]. Oscillometric devices were used because of their ease of use and also to minimise inter-observer variability and digit preference. Although no local validation of Omron M6 was done, a palpation method of blood pressure determination was used in addition for the few children sent to Mulago hospital before recommendations were made upon management.

Participants sat for five minutes before BP measurements were commenced. Three readings were taken at intervals of at least five minutes. For each child, the mean of the three systolic BPs and the mean of the three diastolic BPs were calculated. These readings were compared to internationally-used normograms [11] on the basis of age, gender and height percentile. If the mean systolic or diastolic measurement was $\geq 95$ th percentile for a child on the day 1 visit, then the process was repeated both day 2 and day 3, within one week. HBP was defined as having average mean systolic and/or diastolic readings, taken on all three different days, that were $\geq 95$ th percentile. If the mean systolic and diastolic BPs taken on day 1 were normal (below the $95^{\text {th }}$ percentile) then no further readings were taken.

Children diagnosed with HBP during these initial visits were seen at two subsequent follow-up visits ("follow up 
1 " and "follow up 2") at intervals of approximately three months. $\mathrm{BP}$ readings were repeated three times in one sitting at each follow-up visit. Parents were encouraged to attend the second follow-up visit. Children with mean systolic or diastolic BP $\geq 95^{\text {th }}$ percentile on the three initial visits and at both follow up visits were defined as having sustained HBP and were investigated to identify underlying causes using urine analysis by dipstick, full blood count, renal function tests, chest $\mathrm{x}$-ray and renal ultra sound. Referrals were made to AO at Mulago National Referral Hospital with transport provided.

Two thick blood films were taken from the selected children and prepared on the same slide. The slides were read independently by two trained lab assistants. Stool samples were collected from the same children. Two Kato-Katz slides were prepared from each sample and examined within 30 minutes for hookworm eggs and at a later stage for ova of other species. All children with malaria or helminth infections received treatment and their parents were informed in writing.

We aimed to invite 360 children in rural and 360 children in urban schools to enrol in the study. No preliminary data were available to allow power calculations for the HBP outcome. Data entry and analysis were carried out using EpiInfo version 6 and Stata version 12. The prevalence of HBP based on data from the initial visits was calculated overall, and by each risk factor category. Differences between distribution of risk factors in urban and rural schools were examined using odds ratios and chi-squared tests. Crude associations between each risk factor [age, gender, BMI z-score, family history of high blood pressure, cigarette smoke exposure, rural/urban environment, malaria and helminth infections (analysed by subtype as Schistosoma mansoni or soil-transmitted helminths)] and the outcome of HBP were assessed using logistic regression to calculate odds ratios, 95\% confidence intervals and p-values. Multivariable analysis was then conducted using logistic regression, adjusting for risk factors that were crudely associated with the outcome of interest at $\mathrm{p}<0.2$, and that were not considered to be on the causal pathway between the risk factor of interest and the outcome. Associations between each risk factor and mean systolic blood pressure on "day 1 " and between each risk factor and mean diastolic blood pressure on "day 1" were also investigated, using simple and multiple linear regression, and a similar approach to multivariable analysis as described above.

\section{Results}

Five rural and two urban schools were selected. A total of 381 and 611 children were on the register in selected classes in the rural and urban schools, respectively. The proportion of those registered who enrolled in the study varied between schools ( $24 \%$ to $73 \%$ in rural schools, $48 \%$ to $71 \%$ in urban schools). Lack of enrolment was mainly due to children being absent on the days the study was conducted and to inaccuracies in the class registers. Five hundred and fifty-two students were enrolled (184 from rural and 368 from urban schools, Table 1). They ranged in age from 7 to 18 years with a mean age of 11 years. Two hundred and ninety-six of the participants were female (53.6\%). Children in rural schools, compared to their urban counterparts, were more likely to have malaria (odds ratio (OR) 3.83 (95\% confidence interval 2.08-7.03) $\mathrm{p}<0.001$ ) and to be exposed to cigarette smoke $(1.54(1.01-2.36) \mathrm{p}=0.05)$, but less likely to be infected with schistosomes $(0.24$ (0.08$0.70) \mathrm{p}=0.01$ ) and less likely to be overweight or obese $(\mathrm{p}=0.03)$.

The mean (standard deviation; SD) systolic BP on day 1 was $116.9 \mathrm{mmHg}(12.4 \mathrm{mmHg})$. The mean (SD) diastolic $\mathrm{BP}$ was $68.0 \mathrm{mmHg}(8.7 \mathrm{mmHg})$. On day 1, 94.5\% of the mean systolic results and $84.2 \%$ of the mean diastolic results were above the $50^{\text {th }}$ centile based on internationally used normograms. A similar distribution was seen when children with day $1 \mathrm{BP}$ readings above the 95 percentile were re-measured on subsequent days in the same week (day 2 and day 3; Figure 1). However when children diagnosed with HBP based on days 1, 2 and 3 were seen again at follow up after approximately three and then six months, the distribution of pressures had become more normal: at the final visit, about half of the children seen had pressures below the fiftieth centile, as expected from a normal distribution (Figure 1).

Of the 539 children for whom data were available from the initial visits (days 1-3), 92 (17.1\%, 95\% CI: $13.9 \%$ to $20.2 \%$ ) were found to have HBP. Increasing age (adjusted (a)OR 1.29(1.14-1.47), $\mathrm{p}<0.001$ ), increasing BMI Z-score (aOR $1.70(1.25-2.31), \mathrm{p}=0.001)$ and soil transmitted helminth infection (aOR 2.52 (1.04-6.11), $\mathrm{p}=0.04$ ) were associated with an increased prevalence of HBP based on the initial visits (Table 2). Results analysing mean systolic blood pressure and mean diastolic blood pressure on day 1 as outcomes were similar (Table 3), with increasing age and BMI both strongly positively associated with systolic and diastolic blood pressure. However soil transmitted helminth infection was not associated with either systolic or diastolic blood pressure ( $\mathrm{p}=0.48$ and 0.49 respectively). On the other hand, children infected with malaria had on average lower systolic and diastolic blood pressure than uninfected children. Girls had somewhat higher mean systolic blood pressure than boys, while children from urban schools had reduced mean diastolic blood pressure compared to children from rural schools.

The flow of participants through the study is seen in Figure 2. Of the 92 children with HBP based on the initial set of visits, 74 were present when the schools were visited for follow up visit 1 and 38/74 (51.3\%) still had 
Table 1 Characteristics of study participants

\begin{tabular}{|c|c|c|c|c|}
\hline Variable & Category & Rural schools $(n=184)$ & Urban schools $(n=368)$ & P-value $^{\mathrm{a}}$ \\
\hline \multirow[t]{5}{*}{ Age } & $7-8$ years & $10(5.4 \%)$ & $23(6.3 \%)$ & 0.58 \\
\hline & $9-10$ years & $56(30.4 \%)$ & $129(35.1 \%)$ & \\
\hline & $11-12$ years & $71(38.6 \%)$ & $125(34.0 \%)$ & \\
\hline & 13-14 years & $43(23.4 \%)$ & 77 (20.9\%) & \\
\hline & $15-18$ years & $4(2.2 \%)$ & $14(3.8 \%)$ & \\
\hline \multirow[t]{2}{*}{ Gender } & Male & $94(51.1 \%)$ & $162(44.0 \%)$ & 0.12 \\
\hline & Female & $90(48.9 \%)$ & $206(56.0 \%)$ & \\
\hline \multirow[t]{4}{*}{ BMI-for-age Z-score ${ }^{\text {b }}$} & Underweight & $4(2.2 \%)$ & $6(1.6 \%)$ & 0.03 \\
\hline & Normal & $163(90.1 \%)$ & $298(81.6 \%)$ & \\
\hline & Overweight & $14(7.7 \%)$ & $57(15.6 \%)$ & \\
\hline & Obese & $0(0 \%)$ & $4(1.1 \%)$ & \\
\hline \multirow[t]{3}{*}{ Family history of high blood pressure } & None & $98(53.3 \%)$ & $222(60.3 \%)$ & 0.28 \\
\hline & Any & 67 (36.4\%) & $112(30.4 \%)$ & \\
\hline & Not known & $19(10.3 \%)$ & $34(9.2 \%)$ & \\
\hline \multirow[t]{2}{*}{ Cigarette smoke exposure } & Unexposed & $134(74.0 \%)$ & $295(81.5 \%)$ & 0.05 \\
\hline & Exposed & $47(26.0 \%)$ & $67(18.5 \%)$ & \\
\hline \multirow[t]{2}{*}{ Malaria $^{c}$} & Uninfected & $111(62.7 \%)$ & $103(86.6 \%)$ & $<0.001$ \\
\hline & Infected & 66 (37.3\%) & 16 (13.5\%) & \\
\hline \multirow[t]{2}{*}{ Schistosoma mansoni ${ }^{c}$} & Uninfected & $171(97.2 \%)$ & 99 (89.2\%) & 0.01 \\
\hline & Infected & $5(2.8 \%)$ & $12(10.8 \%)$ & \\
\hline \multirow[t]{2}{*}{ Soil transmitted helminths ${ }^{c}$} & Uninfected & $158(89.8 \%)$ & 98 (89.1\%) & 0.86 \\
\hline & Infected & 18 (10.2\%) & 12 (10.9\%) & \\
\hline
\end{tabular}

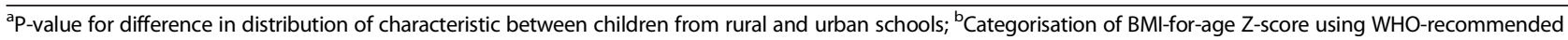
thresholds: underweight (Z-score $<-2)$, normal $(-2 \leq$ Z-score $<1)$, overweight $(1 \leq Z$-score $<2)$, obese (Z-score $\geq 2)$; ${ }^{C}$ Malaria, Schistosoma mansoni and soil transmitted helminth infection status were ascertained for a randomly selected sample of 20 children per class in the urban schools; Missing values in rural and urban school participants, respectively: BMI-for-age Z-score 3, 3; cigarette smoke exposure 3, 6; malaria 7, 1; Schistosoma mansoni 8, 9; soil transmitted helminths 8,10 .

HBP. Thirty-two of these 38 children were seen on follow up visit 2 . Mean systolic and/or diastolic BP remained elevated in $14 / 32$ (43.8\%), thus sustained HBP was confirmed in $2.6 \%(14 / 539 ; 95 \%$ CI: $1.2 \%$ to $4 \%)$ of the original participants. Among the 14 participants, three and eight had diastolic blood pressure above the 95th and 99th percentile, respectively, and three had systolic blood pressure above the 99th percentile. Allowing for losses to follow up and assuming the proportions at each stage of the protocol are representative, we estimate the prevalence of sustained HBP to be $3.8 \%$ (95\% CI: $2.4 \%-5.9 \%$ ).

Children with sustained HBP were invited for review by the paediatricians at Mulago Hospital. Four of the twelve who were reviewed were considered to require treatment. Among children with persistently high blood pressure, urine dipstick, full blood count and creatinine results were normal for all tested. The chest $\mathrm{x}$-ray was abnormal, showing cardiomegaly, for one child. We were unable to identify a secondary cause of hypertension in any of the children with sustained HBP.
Six children who did not meet our criteria for HBP but who had a strong family history of HBP and readings over the $90^{\text {th }}$ centile were also investigated at the referral centre, on the advice of the consultant (AO) (not included in the flow diagram; Figure 2). In one of these children, renal ultrasound demonstrated one kidney smaller than age-appropriate norms. This child subsequently required treatment for HBP.

\section{Discussion}

In this survey among primary school children in Uganda, the initial prevalence of HBP, assessed by measurement in triplicate on three separate days within one week, was high at $17.1 \%$. This is comparable with recent results from adults in Uganda, all from studies in which blood pressure was assessed by two or three repeated measurements on a single day (14.6\% to $22 \%$ ) [12-15]. However, only a minority of our children had sustained HBP on extended follow up. Our estimated prevalence for sustained HBP was 3.8\% (95\% CI: $2.4 \%-5.9 \%$ ). This is in keeping with the figure of 


\section{a}

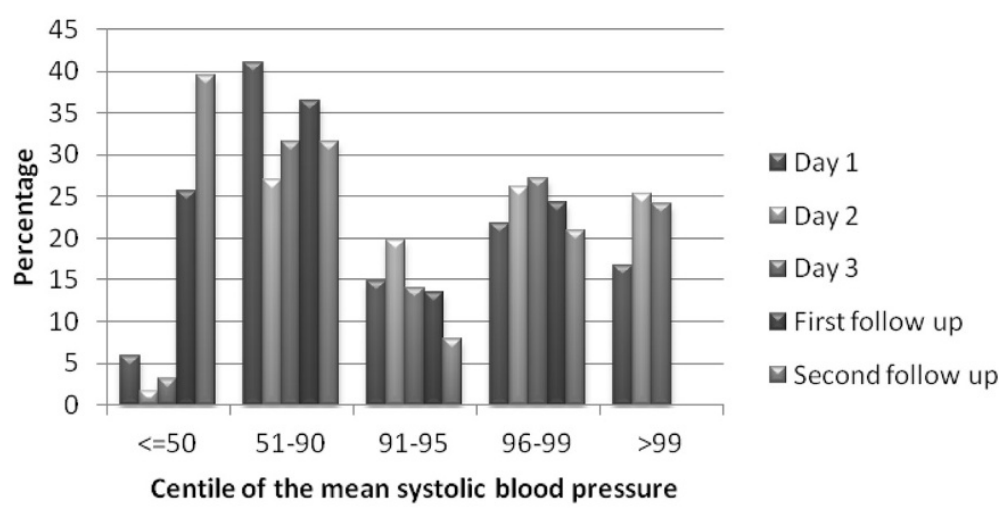

b

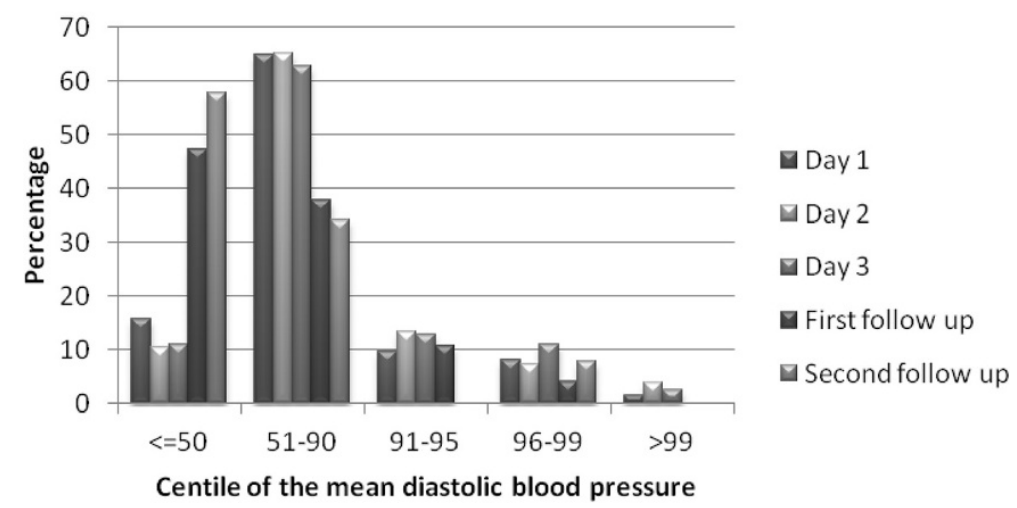

Figure 1 Distribution of normogram-based centiles of the mean systolic (a) and mean diastolic (b) blood pressure readings taken during the initial and follow up visits. The number of children examined at each visit is shown in Figure 2.

$5 \%$ expected to have blood pressure readings above the 95\% percentile. This suggests that, while population-specific normograms might be preferable, the international normograms used may be reasonably applicable in this setting.

The initial negative skew in our data, which resolved on extended follow up, emphasises the importance of repeated measurements over a considerable time period to diagnose HBP in children. Blood pressure varies with circadian rhythms and in response to activity or emotion. These fluctuations are more marked in children (especially around puberty) than in adults [11]. As a result, in order to obtain an accurate picture of a child's blood pressure, it is recommended that repeated measurements are taken at several different sittings on different days [16]. As far as we are aware, ours is the first survey among children in sub-Saharan Africa to have included such extended follow up. Studies from South Africa have determined $\mathrm{BP}$ in children using a protocol similar to our day 1 procedure, with repeated measurements on a single day, and demonstrated HBP prevalence varying between $7.5 \%$ and $22.3 \%$ [6], comparable to our initial estimate of $17.1 \%$. Data from North America collected over a longer period, suggest a prevalence of HBP between $2 \%$ and $5 \%$ [11] comparable to our estimate of sustained HBP of $3.8 \%$.

The initial negative skew also implies that care is needed in interpreting the implications of the associations between risk factors and BP results obtained during the initial three-day testing (in our study) and at a single sitting (in other studies). However, subject to this caveat, in keeping with our results, a study in Zambia found that increasing age was associated with higher blood pressure amongst rural adolescents [17]. We found a weak association with female gender, also, but this was only statistically significant for systolic blood pressure. A limitation of our study was that the stage of puberty was not assessed. Studies from Ghana [18] and Nigeria [19] found that increasing body mass index and urban locality were independently associated with higher blood pressure, but a diagnosis of hypertension was not made. We also designed our study to make rural-urban comparisons, and found some evidence that urban locality was associated with lower diastolic blood pressure. This finding was not replicated for 
Table 2 Factors associated with high blood pressure among school children in Wakiso District, Uganda

\begin{tabular}{|c|c|c|c|c|c|c|}
\hline Variable & & High blood pressure, n (\%) & Crude OR $(95 \% \mathrm{Cl})$ & $\mathrm{P}$ & Adjusted OR $(95 \% \mathrm{Cl})^{\mathrm{b}}$ & $\mathbf{P}$ \\
\hline Age & & & $1.31(1.16,1.49)$ & $<0.001$ & $1.29(1.14,1.47)$ & $<0.001$ \\
\hline \multirow[t]{2}{*}{ Gender } & Male & $35 / 249(14.1 \%)$ & 1 & & 1 & \\
\hline & Female & $57 / 290(19.7 \%)$ & $1.50(0.94,2.37)$ & 0.09 & $1.37(0.85,2.21)$ & 0.20 \\
\hline BMI Z score & & & $1.82(1.34,2.47)^{c}$ & $<0.001$ & $1.70(1.25,2.31)^{c}$ & 0.001 \\
\hline \multirow[t]{3}{*}{ Family history of high blood pressure } & None & $52 / 312(16.7 \%)$ & 1 & 0.47 & - & \\
\hline & Any & $34 / 177(19.2 \%)$ & $1.19(0.74,1.92)$ & & & \\
\hline & Not known & $6 / 50(12.0 \%)$ & $0.68(0.28,1.68)$ & & & \\
\hline \multirow[t]{2}{*}{ Cigarette smoke exposure } & Unexposed & 75/419 (17.9\%) & 1 & & - & \\
\hline & Exposed & $17 / 114(14.9 \%)$ & $0.80(0.45,1.43)$ & 0.45 & & \\
\hline \multirow[t]{2}{*}{ Rural or urban school } & Rural & $30 / 179(16.8 \%)$ & 1 & & - & \\
\hline & Urban & $62 / 360(17.2 \%)$ & $1.03(0.64,1.67)$ & 0.89 & & \\
\hline \multirow[t]{2}{*}{ Malaria $^{\mathrm{a}}$} & Uninfected & $44 / 207(21.3 \%)$ & 1 & & 1 & \\
\hline & Infected & 10/82 (12.2\%) & $0.51(0.25,1.08)$ & 0.08 & $0.63(0.28,1.43)$ & 0.27 \\
\hline \multirow[t]{2}{*}{ Schistosoma mansoni ${ }^{\text {a }}$} & Uninfected & $52 / 263(19.8 \%)$ & 1 & & - & \\
\hline & Infected & 3/17 (117.6\%) & $0.87(0.24,3.14)$ & 0.83 & & \\
\hline \multirow[t]{2}{*}{ Soil transmitted helminths ${ }^{a}$} & Uninfected & 45/249 (18.1\%) & 1 & & 1 & \\
\hline & Infected & $10 / 30(33.3 \%)$ & $2.27(0.99,5.17)$ & 0.05 & $2.52(1.04,6.11)$ & 0.04 \\
\hline
\end{tabular}

${ }^{a}$ Malaria, schistosoma mansoni and soil transmitted helminth infection status were ascertained for a randomly selected sample of 20 children per class in the urban schools; ${ }^{b}$ Odds ratios for age, gender and BMI were adjusted for each other but not for malaria and soil transmitted helminths; odds ratios for malaria and soil transmitted helminths were adjusted for age, gender, BMI and each other; ${ }^{\circ} \mathrm{BMI} Z \mathrm{Z}$ score was analysed as a continuous covariate.

the systolic blood pressure or HBP outcomes, thus it may be a chance finding. Our urban population was sampled from Entebbe and although the children attended urban schools, their levels of poverty meant that, for some, their home lives closely resembled a rural existence (the relatively high prevalence of schistosomiasis in Entebbe children emphasises this). Our rural sample was smaller than intended due to a variety of factors including smaller schools and poor attendance.

We found that malaria infection was associated with lower blood pressure on day 1, although this did not translate to an association with HBP based on the full set of initial visits. To our knowledge, no other study has reported such an association, and this may simply be a reflection of the well known association between clinical malaria and low blood pressure, reflected to a lesser degree in these asymptomatic children. Our finding that soil transmitted helminth infections were associated with increased HBP is surprising (although analysing systolic and diastolic blood pressure on day 1 found no evidence of an association).

Some of these findings may be due to residual confounding: we were unable to account for socioeconomic status in our analysis, and this may be important since a study in Kinshasa, in the Democratic Republic of Congo, showed that elevated blood pressure was associated with low socio-economic status $(\mathrm{OR}=1.2 ; 95 \%$ CI 1.1 to 1.3 ; $\mathrm{p}<0.01)$ [7]. Poverty is generally associated with poor hygiene and increased risk of soil-transmitted helminths and malaria [20]. In future, larger studies among African children would be of interest to determine whether factors associated with HBP on a short-term testing protocol are similar to those associated with sustained HBP, but our sample size was too small for this.

It is interesting to note that most cases of sustained HBP were likely to be primary HBP, even in this population with little obesity and generally high levels of physical activity. This may be the result of genetics or of nutritional factors such as high salt consumption that were not determined in this study: further investigation is needed. Although it can be difficult to assess heart size using $\mathrm{x}$-rays in children, the report of cardiomegaly on chest $\mathrm{x}$-ray in one case suggests that HBP may have been present for a sufficient period, and at sufficient intensity, to result in cardiac strain.

Identification of children with HBP in this setting was a relatively easy step. However, provision of follow up and treatment presents a challenge. For us, investigation proved difficult to arrange despite the fact that it was offered free by the research programme. None of the parents of the four children identified as requiring treatment anticipated that they would be able to afford the prescribed drugs.

According to Ministry of Education data, only $68.4 \%$ of eligible children in Wakiso district were enrolled in primary schools at the time of this study [21], and outof-school children were not sampled. At the opposite extreme, children at private schools were not sampled 
Table 3 Factors associated with systolic and diastolic blood pressure among school children in Wakiso District, Uganda

\begin{tabular}{|c|c|c|c|c|c|c|}
\hline Variable & & Mean (SD) & $\begin{array}{l}\text { Mean difference } \\
(95 \% \mathrm{Cl})\end{array}$ & $\mathbf{P}$ & $\begin{array}{l}\text { Adjusted mean difference } \\
(95 \% \mathrm{Cl})^{\mathrm{b}}\end{array}$ & $\mathbf{P}$ \\
\hline \multicolumn{7}{|l|}{ Systolic blood pressure } \\
\hline Age & & & $3.24(2.73,3.75)$ & $<0.001$ & $3.14(2.63,3.65)$ & $<0.001$ \\
\hline \multirow[t]{2}{*}{ Gender } & Male & $115.9(12.5)$ & reference & & reference & \\
\hline & Female & $117.7(12.3)$ & $1.79(-0.28,3.87)$ & 0.09 & $1.81(0.01,3.61)$ & 0.05 \\
\hline BMI Z score & & & $3.29(2.14,4.43)^{c}$ & $<0.001$ & $2.78(1.74,3.83)^{c}$ & $<0.001$ \\
\hline \multirow[t]{3}{*}{ Family history of high blood pressure } & None & $116.5(12.7)$ & reference & 0.13 & reference & 0.97 \\
\hline & Any & $118.0(11.9)$ & $1.49(-0.76,3.75)$ & & $0.25(-1.71,2.21)$ & \\
\hline & Not known & $114.2(11.2)$ & $-2.34(-6.01,1.34)$ & & $-0.01(-3.19,3.18)$ & \\
\hline \multirow[t]{2}{*}{ Cigarette smoke exposure } & Unexposed & $117.4(12.2)$ & reference & & reference & \\
\hline & Exposed & $114.7(12.6)$ & $-2.68(-5.22,-0.13)$ & 0.04 & $-1.03(-3.25,1.18)$ & 0.36 \\
\hline \multirow[t]{2}{*}{ Rural or urban school } & Rural & $116.8(10.8)$ & reference & & & \\
\hline & Urban & $117.0(13.1)$ & $0.22(-1.98,2.42)$ & 0.84 & & \\
\hline \multirow[t]{2}{*}{ Malaria $^{a}$} & Uninfected & $119.1(12.0)$ & reference & & reference & \\
\hline & Infected & $113.7(1.06)$ & $-5.40(-8.37,-2.43)$ & $<0.001$ & $-3.79(-6.31,-1.27)$ & 0.003 \\
\hline \multirow[t]{2}{*}{ Schistosoma mansoni ${ }^{a}$} & Uninfected & $117.8(11.8)$ & reference & & & \\
\hline & Infected & $115.8(13.3)$ & $-2.05(-7.91,3.80)$ & 0.49 & & \\
\hline \multirow[t]{2}{*}{ Soil transmitted helminths ${ }^{\mathrm{a}}$} & Uninfected & $117.4(12.0)$ & reference & & & \\
\hline & Infected & $120.0(10.6)$ & $2.52(-2.00,7.04)$ & 0.27 & & \\
\hline \multicolumn{7}{|l|}{ Diastolic blood pressure } \\
\hline Age & & & $1.53(1.14,1.91)$ & $<0.001$ & $1.48(1.10,1.85)$ & $<0.001$ \\
\hline \multirow[t]{2}{*}{ Gender } & Male & $67.6(8.9)$ & reference & & reference & \\
\hline & Female & $68.3(8.5)$ & $0.73(-0.74,2.19)$ & 0.33 & $0.86(-0.50,2.23)$ & 0.22 \\
\hline BMI Z score & & & $1.56(0.73,2.38)$ & $<0.001$ & $1.64(0.85,2.43)$ & $<0.001$ \\
\hline \multirow[t]{3}{*}{ Family history of high blood pressure } & None & $67.5(9.2)$ & reference & 0.20 & & \\
\hline & Any & $68.8(8.1)$ & $1.39(-0.20,2.98)$ & & & \\
\hline & Not known & $67.3(7.3)$ & $-0.14(-2.74,2.45)$ & & & \\
\hline \multirow[t]{2}{*}{ Cigarette smoke exposure } & Unexposed & $68.0(8.5)$ & reference & & & \\
\hline & Exposed & $67.6(9.1)$ & $-0.39(-2.18,1.40)$ & 0.67 & & \\
\hline \multirow[t]{2}{*}{ Rural or urban school } & Rural & $69.8(7.8)$ & reference & & reference & \\
\hline & Urban & $67.1(9.0)$ & $-2.73(-4.26,-1.20)$ & $<0.001$ & $-3.08(-4.54,-1.62)$ & $<0.001$ \\
\hline \multirow[t]{2}{*}{ Malaria $^{a}$} & Uninfected & $69.8(8.7)$ & reference & & reference & \\
\hline & Infected & $66.9(8.2)$ & $-2.93(-5.11,-0.74)$ & 0.009 & $-2.84(-4.97,-0.72)$ & 0.009 \\
\hline \multirow[t]{2}{*}{ Schistosoma mansoni ${ }^{a}$} & Uninfected & $69.3(8.6)$ & reference & & & \\
\hline & Infected & $67.8(9.7)$ & $-1.52(-5.78,2.75)$ & 0.48 & & \\
\hline \multirow[t]{2}{*}{ Soil transmitted helminths ${ }^{a}$} & Uninfected & $69.1(8.8)$ & reference & & & \\
\hline & Infected & $70.9(6.9)$ & $1.80(-1.49,5.09)$ & 0.28 & & \\
\hline
\end{tabular}

${ }^{a}$ Malaria, Schistosoma mansoni and soil transmitted helminths infection status were ascertained for a randomly selected sample of 20 children per class in the urban schools; ${ }^{b}$ For associations with systolic blood pressure, mean differences for age, gender, BMI and cigarette smoke exposure were adjusted for each other but not for malaria; mean difference for malaria was adjusted for age, gender, BMI and cigarette smoke exposure. For associations with diastolic blood pressure, mean differences for age, gender, BMI and rural/urban school were adjusted for each other but not for malaria; mean difference for malaria was adjusted for age, gender, BMI and rural/urban school; ' $\mathrm{BMI} Z \mathrm{Z}$ score was analysed as a continuous covariate.

and these may, at present, be the group most at risk from NCD risk factors associated with changing lifestyles. These two groups need to be considered in future studies.

\section{Conclusion}

There is a lack of information about the prevalence of HBP and of the risk factors leading to HBP in tropical sub-Saharan Africa. While studies in adults suggest a 


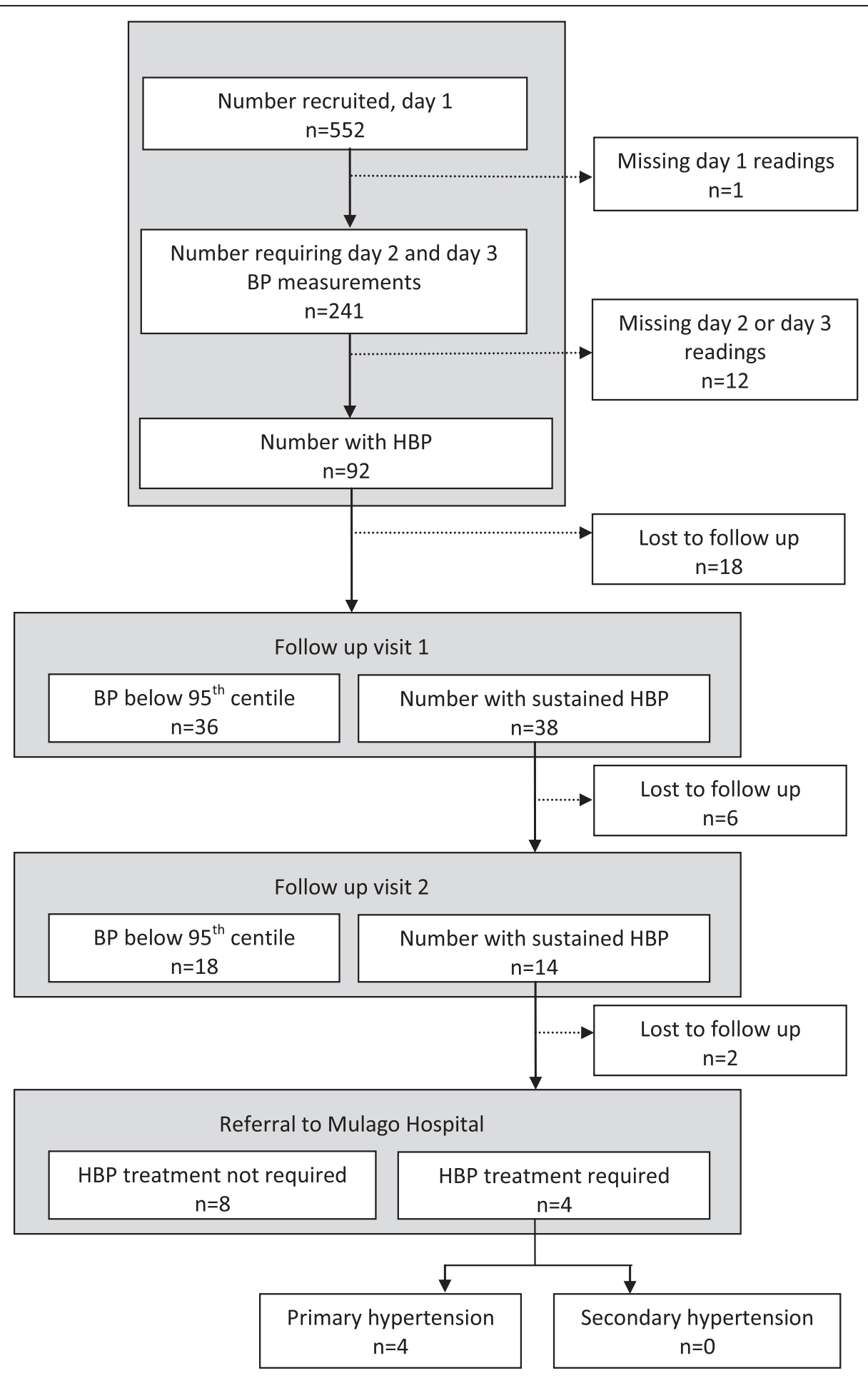

Figure 2 The flow of participants through the blood pressure protocol procedures.

high prevalence of hypertension, this study among children, with extended follow up, suggested a prevalence of HBP in keeping with international norms. However, a less meticulous study, confined to a shortterm, three-day protocol for BP assessment, would have resulted in a major over-estimate of $\mathrm{HBP}$ in this population. School-based surveys for NCD risks are feasible and informative in this setting, but rigorous protocols need to be followed for the assessment of HBP in children. 


\section{Competing interests}

The authors declare that they have no competing interests.

\section{Authors' contributions}

FK designed and led the study under the supervision of ELW and AME. FK and ELW conducted the analyses. FK, ELW and AME drafted the manuscript $\mathrm{DR}, \mathrm{SAL}$ and $\mathrm{AO}$ contributed to follow up and care of participants, with $\mathrm{AO}$ contributing expert advice on referral procedures, investigation and management. MK contributed to field work and follow up. All authors reviewed the manuscript. All authors read and approved the final manuscript.

\section{Acknowledgements}

We thank participants and school staff, and all members of the field and clinic team, and the laboratory staff at the MRC/UVRI Uganda Research Unit on AIDS

Financial support was received from the London School of Hygiene and Tropical Medicine, and from Prof Elliott's Wellcome Trust senior fellowship, grant number 079110. Dr Webb was supported in part by the UK Medical Research Council.

The funders had no role in study design, in the collection, analysis, and interpretation of data, in the writing of the manuscript or in the decision to submit the manuscript for publication.

\section{Author details}

${ }^{1}$ London School of Hygiene and Tropical Medicine, Keppel Street, London WC1E 7HT, UK. ${ }^{2}$ MRC/UVRI Uganda Research Unit on AIDS, P.O. Box 49, Entebbe, Uganda. ${ }^{3}$ Department of Paediatrics \& Child Health, Makerere University, College of Health Sciences, P.O BOX 7072, Kampala, Uganda. ${ }^{4}$ Current address: The Surgery @ Aylestone, 672 Aylestone Road, Leicester LE2 8PR, UK. ${ }^{5}$ Current address: St Mary's Family Clinic, P.O.Box 23610 Kampala, Uganda. ${ }^{6}$ Current address: Department of Paediatrics \& Child Health; Faculty of Medicine, University of Rwanda, P.O. BOX 117, Butare, Rwanda.

Received: 2 September 2014 Accepted: 17 November 2014 Published: 26 November 2014

\section{References}

1. Prevention and control of non-communicable diseases. [www.un.org/en/ ga/ncdmeeting2011/]

2. WHO: WHO Global Burden of Disease, 2004 Update. ; 2004. http://www.who. int/healthinfo/global_burden_disease/2004_report_update/en/, accessed 20th November 2014

3. Kearney PM, Whelton M, Reynolds K, Muntner P, Whelton PK, He J: Global burden of hypertension: analysis of worldwide data. Lancet 2005, 365(9455):217-223.

4. Berenson GS, Srinivasan SR, Bao W, Newman WP, Tracy RE, Wattigney WA: Association between multiple cardiovascular risk factors and atherosclerosis in children and young adults. N Engl J Med 1998, 338(23):1650-1656.

5. Lauer RM, Clarke WR: Childhood risk factors for high adult blood pressure: the Muscatine study. Pediatrics 1989, 84(4):633-641.

6. Monyeki $\mathrm{K}$, Kemper $\mathrm{H}$ : The risk factors for elevated blood pressure and how to address cardiovascular risk factors: a review in paediatric populations. J Hum Hypertens 2008, 22(7):450-459.

7. Longo-Mbenza B, Lukoki Luila E, M'Buyamba-Kabangu JR: Nutritional status, socio-economic status, heart rate, and blood pressure in African school children and adolescents. Int J Cardiol 2007, 121(2):171-177.

8. Hansen ML, Gunn PW, Kaelber DC: Underdiagnosis of hypertension in children and adolescents. JAMA 2007, 298(8):874-879.

9. Uganda National Household Survey Report 2009/2010. In Uganda Bureau of Statistics; http://www.ubos.org/UNHS0910/unhs200910.pdf, accessed 20th November 2014

10. O'Brien $E$, Asmar R, Beilin L, Imai Y, Mancia G, Mengden T, Myers M, Padfield P, Palatini P, Parati G, Pickering T, Redon J, Staessen J, Stergiou G, Verdecchia P, European Society of Hypertension Working Group on Blood Pressure Monitoring: Practice guidelines of the European society of hypertension for clinic, ambulatory and self blood pressure measurement. J Hypertens 2005. 23:697-701.

11. National High Blood Pressure Education Program Working Group on High Blood Pressure in Children and Adolescents: The fourth report on the diagnosis, evaluation, and treatment of high blood pressure in children and adolescents. Pediatrics 2004, 114:555-576.

12. Musinguzi G, Van Geertruyden JP, Bastiaens H, Nuwaha F: Uncontrolled hypertension in Uganda: a comparative cross-sectional study. J Clin Hypertens 2014, doi:10.1111/jch.12371. [Epub ahead of print].

13. Kotwani P, Kwarisiima D, Clark TD, Kabami J, Geng EH, Jain V, Chamie G, Petersen ML, Thirumurthy H, Kamya MR, Charlebois ED, Havlir DV, SEARCH Collaboration: Epidemiology and awareness of hypertension in a rural Ugandan community: a cross-sectional study. BMC Public Health 2013, 13:1151.

14. Murphy GA, Asiki G, Ekoru K, Nsubuga RN, Nakiyingi-Miiro J, Young EH, Seeley J, Sandhu MS, Kamali A: Sociodemographic distribution of non-communicable disease risk factors in rural Uganda: a cross-sectional study. Int J Epidemiol 2013, 42(6):1740-1753.

15. Mondo CK, Otim MA, Akol G, Musoke R, Orem J: The prevalence and distribution of non-communicable diseases and their risk factors in Kasese district, Uganda. Cardiovasc J Afr 2013, 24(3):52-57.

16. McCrindle BW: Assessment and management of hypertension in children and adolescents. Nat Rev Cardiol 2010, 7(3):155-163.

17. Ng'andu $\mathrm{NH}$ : Blood pressure levels of Zambian rural adolescents and their relationship to age, sex, weight, height and three weight-for-height indices. Int J Epidemiol 1992, 21(2):246-252.

18. Agyemang C, Redekop WK, Owusu-Dabo E, Bruijnzeels MA: Blood pressure patterns in rural, semi-urban and urban children in the Ashanti region of Ghana, West Africa. BMC Public Health 2005, 5:114

19. Ejike CE, Ugwu CE, Ezeanyika LU, Olayemi AT: Blood pressure patterns in relation to geographic area of residence: a cross-sectional study of adolescents in Kogi state, Nigeria. BMC Public Health 2008, 8:411.

20. Cooper PJ, Barreto ML, Rodrigues LC: Human allergy and geohelminth infections: a review of the literature and a proposed conceptual model to guide the investigation of possible causal associations. Br Med Bull 2006, 79-80(1):203-218.

21. Ministry of Education and Sports for the Republic of Uganda. In 2010. http://www.education.go.ug/

doi:10.1186/1471-2458-14-1223

Cite this article as: Kidy et al:: Blood pressure in primary school children in Uganda: a cross-sectional survey. BMC Public Health 2014 14:1223.

\section{Submit your next manuscript to BioMed Central and take full advantage of:}

- Convenient online submission

- Thorough peer review

- No space constraints or color figure charges

- Immediate publication on acceptance

- Inclusion in PubMed, CAS, Scopus and Google Scholar

- Research which is freely available for redistribution 